\title{
The Combination of Equipment Scale and Feature Scale Models for Chemical Vapor Deposition Via a Homogenization Technique
}

\author{
MATTHIAS K. GOBBERT ${ }^{\mathrm{a}^{*}}$, TIMOTHY S. CALE ${ }^{\mathrm{b} \dot{\dagger}}$ and CHRISTIAN A. RINGHOFER ${ }^{\mathrm{a} \dot{+}}$ \\ ${ }^{\mathrm{a}}$ Department of Mathematics, Arizona State University, Tempe, AZ $85287-1804,{ }^{\mathrm{b}}$ Center for Solid State Electronics Research, Arizona State \\ University, Tempe, AZ 85287-6206
}

\begin{abstract}
In the context of semiconductor manufacturing, chemical vapor deposition (CVD) denotes the deposition of a solid from gaseous species via chemical reactions on the wafer surface. In order to obtain a realistic process model, this paper proposes the introduction of an intermediate scale model on the scale of a die. Its mathematical model is a reaction-diffusion equation with associated boundary conditions including a flux condition at the micro structured surface. The surface is given in general parameterized form. A homoganization technique from asymptotic analysis is used to replace this boundary condition by a condition on the flat surface to make a numerical solution feasible. Results from a mathematical test problem are included.
\end{abstract}

Keywords: Homogenization, Asymptotic Analysis, Finite Differences, Partial Differential Equations , Chemical Vapor Deposition, Chemical Engineering

\section{INTRODUCTION}

To model chemical vapor deposition (CVD) in single wafer reactors, attempts have been made at linking reactor scale models (RSM) and feature scale models (FSM) to obtain realistic simulation results [1]. In these studies, reactor scale predictions have been used as inputs to feature scale models, but no information was fed back from the feature scale to the reactor scale. But features are typically arranged in clusters, which remains unaccounted for in this approach. Also, any direct combination of these models must suffer from the vast differences in length scales between the reactor scale $\left(10^{-1} \mathrm{~m}\right)$ and the feature scale $\left(10^{-6} \mathrm{~m}\right)$.

Therefore, we propose the introduction of a mesoscopic scale model (MSM) on the scale of a die to remedy these problems. A schematic is shown in Figure 1. To obtain an integrated process simulator, MSMs encompassing several clusters of features each are introduced at several positions on the wafer bridging the length scale differences between the reactor scale and the feature scale. By encompassing several feature clusters, a MSM also accounts for the effects of varying density of feature clustering and of cluster spacing. For the study of these feature-to-feature

\footnotetext{
* Present address : Department of Mathematics and Statistics, University of Maryland, Baltimore, MD 21250. Fax : (410) $455-1066$ Email : gobbert@math.umbc.edu

$\dagger$ Fax : (602) 965-8118. Email : cale@enws165.eas.asu.edu

$\doteqdot$ Fax : (602) 965-8119. Email : ringhofer@asu.edu
} 


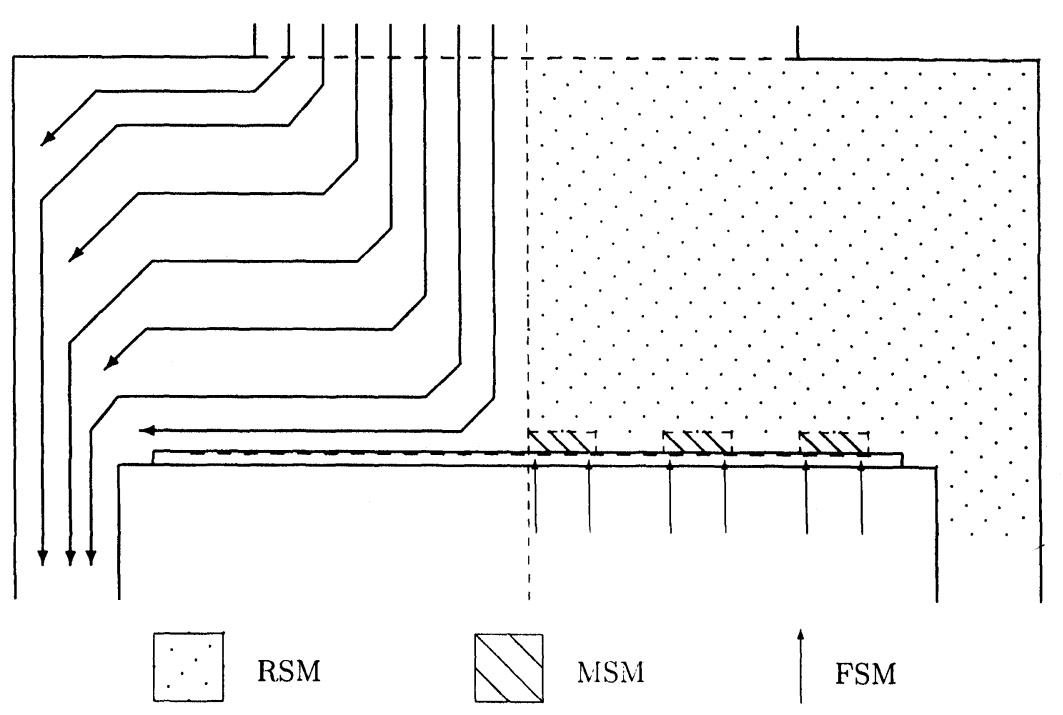

FIGURE 1 Schematic of the reactor with flow pattern and typical domains of the models

effects, the MSM can also be used in a stand-alone mode.

Mathematically, the domain of the MSM is comprised of the gas phase just above one die on the wafer surface. Assuming that the pressure is sufficiently high, the model consists of a reaction-diffusion equation with associated boundary conditions for each chemical species in the gas domain; specifically at the wafer surface, a flux condition is imposed. It is this boundary condition that makes the problem numerically challenging, since it is impossible even for a die scale model to accurately resolve features on the scale of the micro structure.

We solved this problem using a homogenization technique from asymptotic analysis, which allows for the replacement of the micro structured surface by a flat surface by taking into account the increase in surface area inside the feature clusters. The mathematical derivation for surfaces that can be expressed in functional form has been given in [3]. This paper extends the derivation to arbitrary surfaces in parameterized form, which allows for instance for overhangs at the sides of the features.

\section{DERIVATION}

Mathematically, the problem is given as a reactiondiffusion equation with associated boundary conditions. After a suitable non-dimensionalization procedure, the dimensionless concentration $u(x, y)$ has to satisfy the differential equation

$$
\frac{\partial u}{\partial t}=\operatorname{div}_{x y}\left(D(u, x, y) \nabla_{x y} u\right)+R_{g}(u, x, y)
$$

as well as the boundary conditions

$$
\begin{gathered}
-e_{1}^{T}\left(-D \nabla_{x y} u\right)=0 \quad x=0, \\
e_{1}^{T}\left(-D \nabla_{x y} u\right)=0 \quad x=X, \\
u=c^{t o p}(x) \quad y=Y, \\
v^{T}\left(-D \nabla_{x y} u\right)=S(u, x, y,) \quad(x, y) \in \Gamma_{w} .
\end{gathered}
$$

$D$ is a symmetric positive definite diffusivity matrix and $e_{1}=(1,0)^{T}$ the first unit vector.

$\Gamma_{w}$ denotes the parameterized wafer surface and $v$ the outer unit normal vector on $\Gamma_{w}$.

The wafer surface $\Gamma_{w}$ is parameterized with the macroscopic variable $s$ as

$$
(x, y)=(s+\varepsilon \alpha(s, s / \varepsilon), \varepsilon \beta(s, s / \varepsilon)) \quad 0 \leq s \leq 1 .
$$


In a fully periodic surface, the surface would be assumed to be periodic in $s$ with period $\varepsilon$, where $0<\varepsilon<<1$ is a "small" quantity. However, the surface varies on both the macro scale as well as the micro scale; this fact is explicitly modeled by the dependence on the slowly changing (macroscopic) variable $s$ and the fast changing (microscopic) variable $\sigma=s / \varepsilon$, respectively. In the definition of $x, \varepsilon \alpha(\mathrm{s}, \sigma)$ represents then a microscopic perturbation of $s$. In $y, \varepsilon \beta(s, \sigma)$ models the microscopic surface height depending on both the macroscopic and microscopic parameterization. This parameterization allows for instance for overhangs in the surface structure. Since the surface can clearly be very different from one region to another (macroscopically), periodicity is only assumed in the fast changing variable $\sigma$ (microscopically).

Hence, all surface related quantities are assumed to be periodic with period 1 in the fast changing variable, in particular the surface variables $\alpha$ and $\beta$ satisfy

$$
\begin{array}{r}
\alpha(s, \sigma+1)=\alpha(s, \sigma), \quad \beta(s, \sigma+1)=\beta(s, \sigma) \\
\text { for all } 0 \leq s \leq 1 .
\end{array}
$$

This really means that adjacent features are assumed to be identical, while distant features can be different. It is assumed that the parameterization is welldefined.

The idea behind the homogenization technique to be used is the elimination of dependence on the microscopic parameter $\sigma$. To this end, the surface representation is formally inflated to three dimensions depending on the two parameters $\mathrm{s}$ and a independently, that is no relationship between $s$ and $\sigma$ is assumed now. This results in a three-dimensional representation of the wafer surface $\tilde{\Gamma}_{w}$, parameterized by $s$ and $\sigma$ independently:

$$
\begin{aligned}
&(x, y, \xi)=(s+\varepsilon \alpha(\sigma), \varepsilon \beta(s, \sigma), \sigma+\alpha(\sigma)) \\
& 0 \leq s \leq 1,0 \leq \sigma \leq 1 / \varepsilon .
\end{aligned}
$$

For this surface, a homogenization technique like in [3] is used to find the appropriate problem for the leading term of the bulk solution $\tilde{w}_{0}$ :

$$
\frac{\partial \tilde{w}_{0}}{\partial t}=\operatorname{div}_{x y}\left(D\left(\tilde{w}_{0}, x, y\right) \nabla_{x y} \tilde{w}_{0}\right)+R_{g}\left(\tilde{w}_{0}, x, y\right)
$$

with the boundary conditions

$$
\begin{gathered}
-e_{1}^{T}\left(-D \nabla_{x y} \tilde{w}_{0}\right)=0 \quad \text { at } x=0, \\
e_{1}^{T}\left(-D \nabla_{x y} \tilde{w}_{0}\right)=0 \quad \text { at } x=X, \\
\tilde{w}_{0}=c^{t o p}(x) \quad \text { at } y=Y, \\
-e_{2}^{T}\left(-D \nabla_{x y} \tilde{w}_{0}\right)=\tilde{\sigma} S \quad \text { at } y=0,
\end{gathered}
$$

where $\tilde{\sigma}$ is given by

$$
\tilde{\sigma}=\int_{0}^{1} \sqrt{\left(1+\frac{\partial \alpha}{\partial \sigma}\right)^{2}+\left(\frac{\partial \beta}{\partial \sigma}\right)^{2}} d \sigma .
$$

This is the simplified problem on a rectangular domain that can be efficiently solved by numerical methods. The key is that the effect of the micro structured surface is summarized into a macroscopic correction factor in the flux condition at the wafer surface.

\section{NUMERICAL DEMONSTRATION}

To demonstrate the method, a mathematical test problem has been solved with the dimensionless parameters $\epsilon$ chosen sufficiently large to allow for a classic solution by full resolution of the surface. First, the results denoted by the solid lines in Figures 2 and 3 are obtained from a solution of the simplified problem (9)-(13). Second, the dotted lines represent the solution obtained by solving the original problem (1)-(5) after transforming its domain onto a rectangle.

The problem uses $\varepsilon=1 / 16$ and the surface function $y=\tilde{h}(x)=\varepsilon h(x, x / \epsilon), h(x, \xi)=4 x(1-x) \sin \left(\omega_{0} \xi\right)$ with $\omega_{0}=32 \pi \varepsilon$ on the unit square, hence $X=Y=1$.

Figure 2 shows the concentration levels throughout the domain. They clearly agree everywhere except in a region close to the surface, where oscillations are introduced by actually resolving the surface structure. Figure 3 shows that the net flux into the surface predicted by the asymptotic solution captures the average of the true flux. Both facts demonstrate that the 


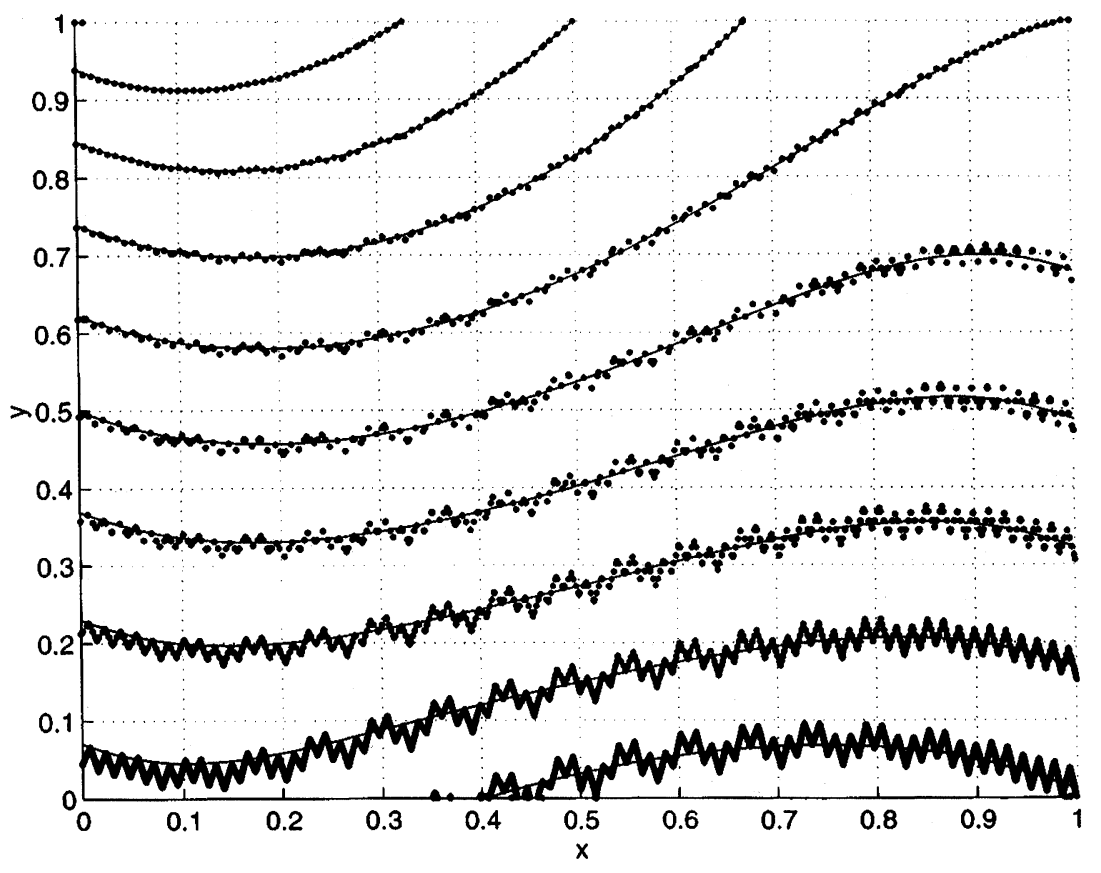

FIGURE 2 Contour plot of solutions

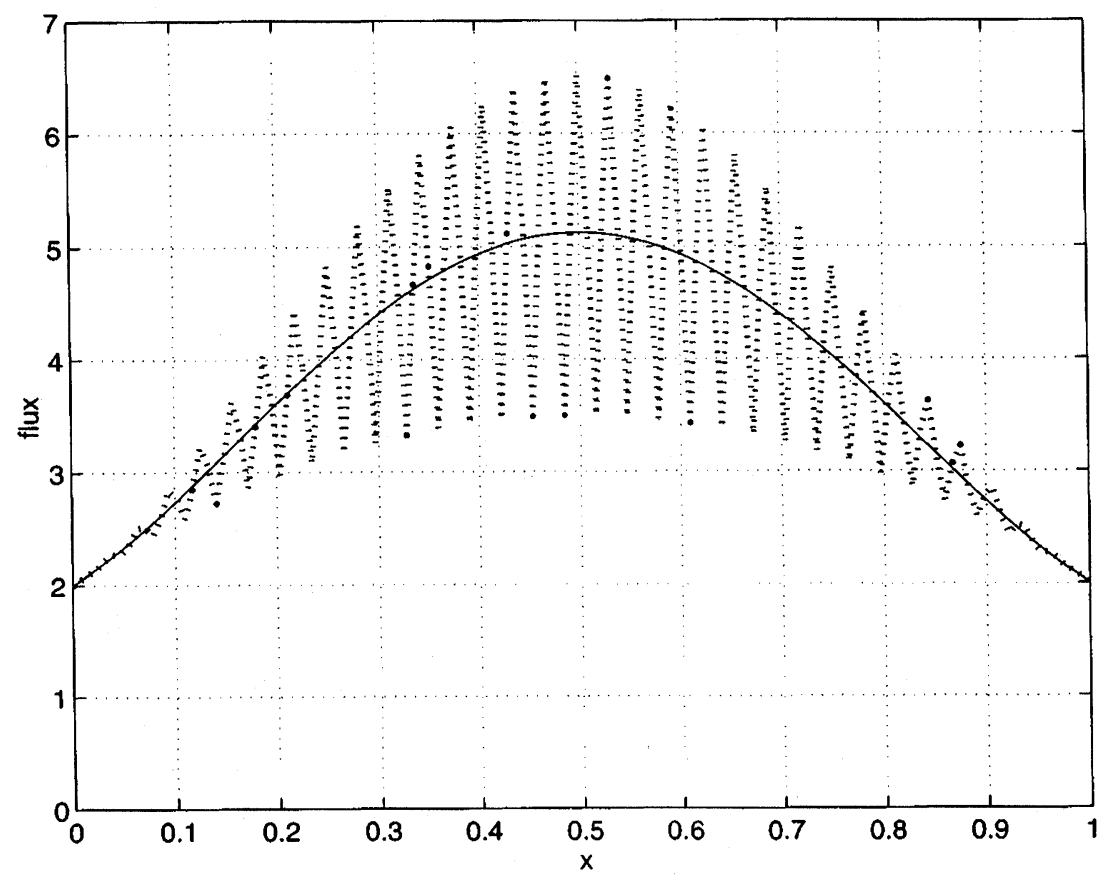

FIGURE 3 Flux plot of solutions 
method is capable of modeling the quantities relevant to the interfaces with the reactor scale model (via the concentration levels near the top) and the feature scale model (via the average flux).

A demonstration of the capability of the mesoscopic scale model to study feature-to-feature effects for a physical example is contained in [2].

\section{Acknowledgements}

This work was partially supported by ARPA Grant No. F49620-93-1-0062 and Semiconductor Research Corporation contract SJ-174.

\section{References}

[1] T. S. Cale, J.-H. Park, T. H. Gandy, G. B. Raupp, and M. K. Jain. "Step coverage predictions using combined reactor scale and feature scale models for blanket tungsten LPCVD." Chemical Engineering Communications, 119:197-220, 1993.

[2] M. K. Gobbert, T. S. Cale, and C. A. Ringhofer. "One approach to combining equipment scale and feature scale models." In M. Meyyappan, D. J. Economou, and S. W. Butler, editors, Process Control, Diagnostics, and Modeling in Semiconductor Manufacturing, pages 553-563, Reno, NV, May 1995. Electrochemical Society, 187th Meeting.
[3] M. K. Gobbert and C. A. Ringhofer. "An asymptotic analysis for a model of chemical vapor deposition on a micro structured surface." SIAM Journal on Applied Mathematics, accepted.

\section{Biographies}

Matthias K. Gobbert is a graduate student in the Department of Mathematics at Arizona State University. He is working on the numerical solution of partial differential equations arising in semiconductor process modeling.

Timothy S. Cale is a professor of Chemical Engineering and Interim Director of the Center for Solid State Electronics Research at Arizona State University. His research combines experiments with modeling and simulations to develop fundamental understanding of transport and reaction phenomena.

Christian A. Ringhofer is a professor of Mathematics at Arizona State University. His work includes asymptotic and numerical methods for the solution of partial differential equations. 

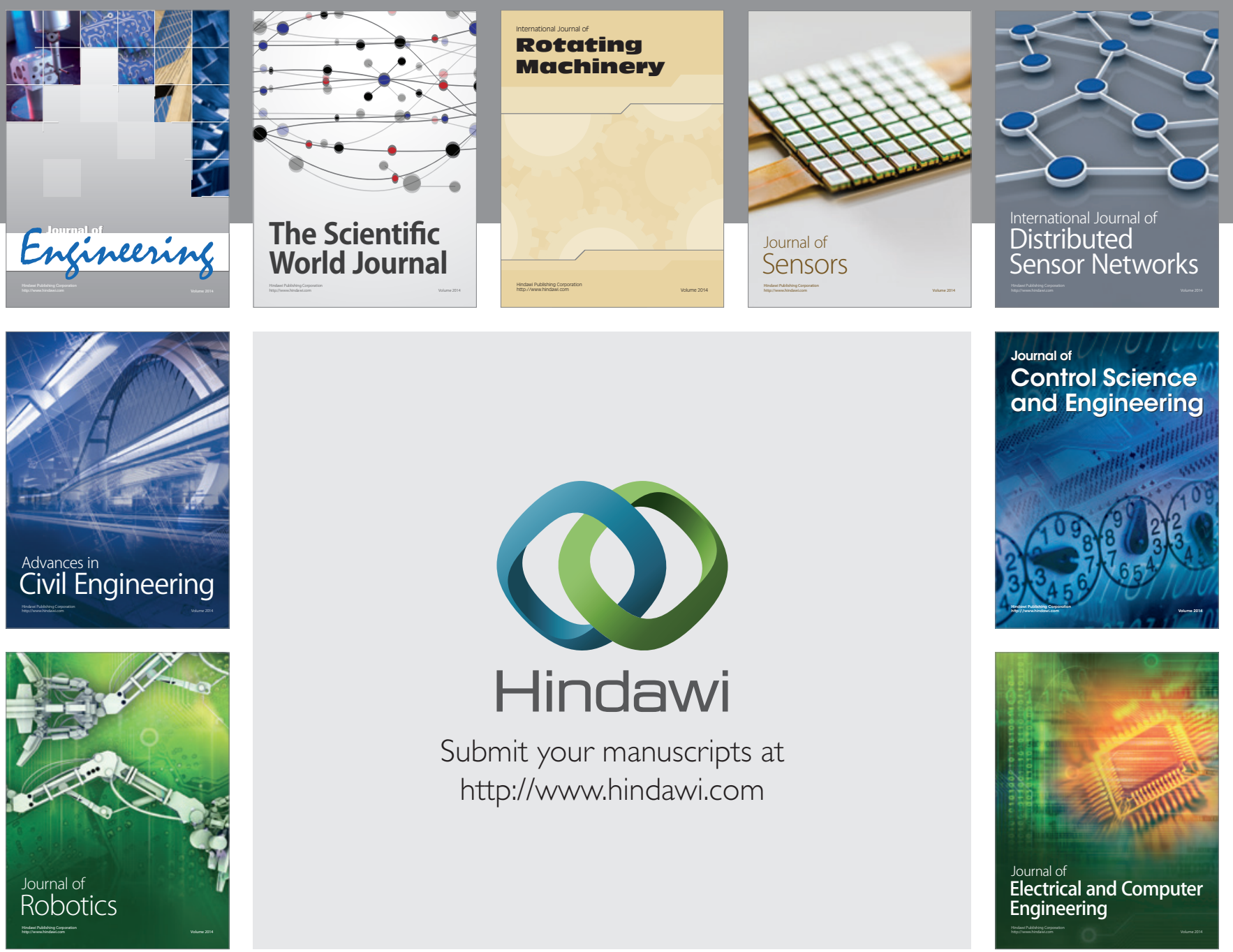

Submit your manuscripts at

http://www.hindawi.com
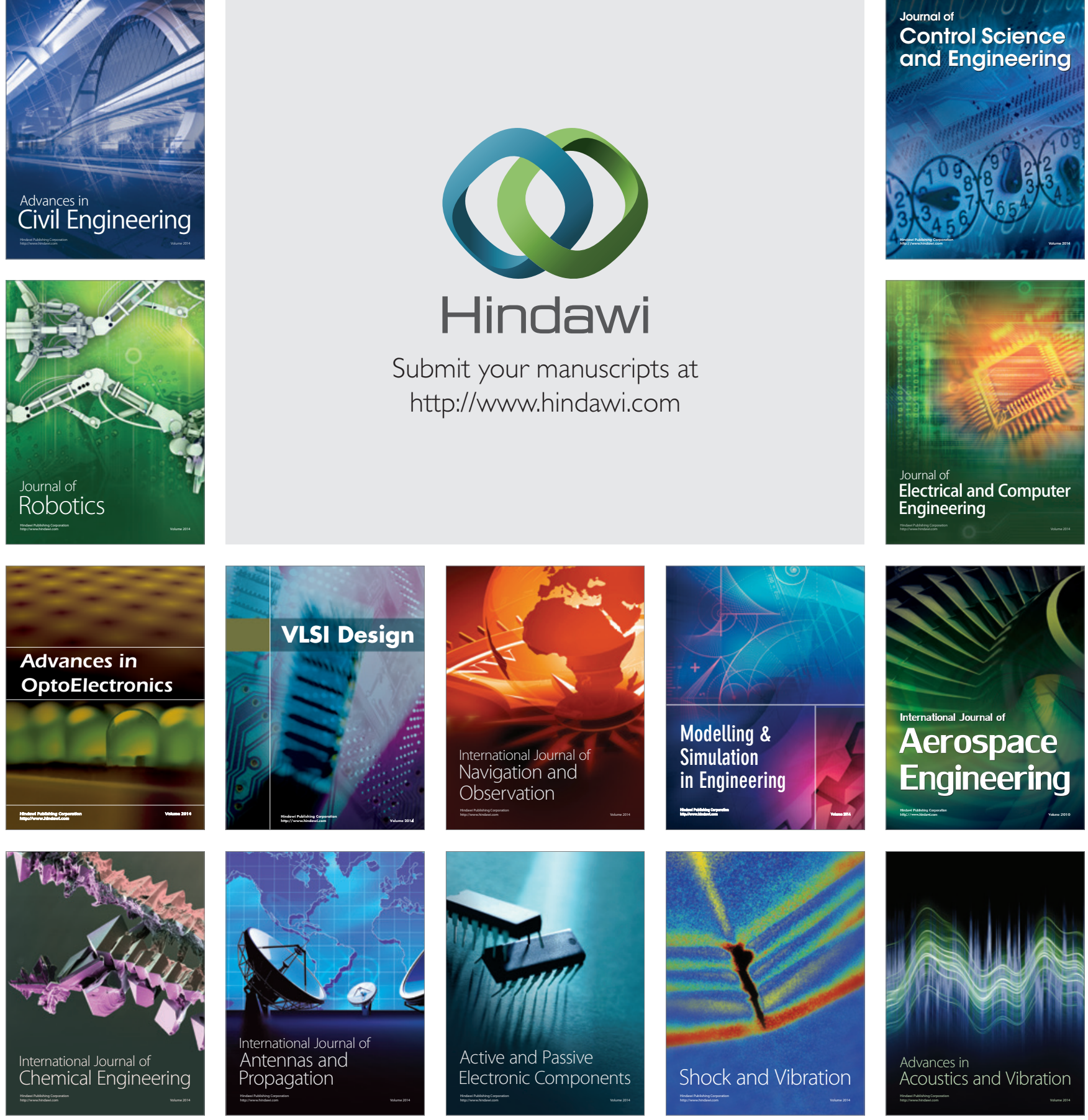